\title{
Post-transcriptional control of c-erb B-2 overexpression in stomach cancer cells
}

\author{
Chang-Dae Bae ${ }^{1}$, Yong-Sung Juhnn ${ }^{2}$ and \\ Joo-Bae Park ${ }^{1,3}$ \\ ${ }^{1}$ Department of Molecular Cell Biology, Sungkyunkwan University \\ School of Medicine, Suwon 440-746, Kyungkido, Korea \\ ${ }^{2}$ Department of Biochemistry and Molecular Biology, Seoul National \\ University College of Medicine, Seoul 110-799, Korea \\ ${ }^{3}$ Corresponding author: Tel, +82-31-299-6130; \\ Fax, +82-31-299-6149; E-mail, jbpark@med.skku.ac.kr
}

Accepted 26 February, 2001

Abbreviations: UTR, untranslated region; Met, methionine; FBS, fetal bovine

\begin{abstract}
The growth factor receptor oncogene, c-erb B-2, is frequently overexpressed in the adenocarcinomas of breast, ovary, lung and stomach. Although the mechanism of erb B-2 overexpression is thought as the result of transcriptional upregulation in many primary human carcinomas, expression rate of c-erb B-2 at mRNA level is usually lower than the level of translated protein. We also found that the expression of erb B-2 in SNU-1 stomach cancer cells was greater at post-transcription level (Bae et al., 1993). To explore the underlying mechanism of erb B-2 protein overexpression, we have chosen two cells lines, SNU-1 and SNU-16 where transcription rate of erb B2 was closely resemble to each other while expressed protein levels were quite different. The synthesis rate of erb B-2 protein in SNU-1 cells was faster than SNU-16 cells while levels of erb B-2 mRNA were found to be similar in both cell lines. The half-life of the expressed erb B-2 protein was not significantly different in both cell lines. Analysis of the 5' untranslated region (UTR) of erb B-2 mRNA (-1 -323) showed no sequence abnormality in both cell lines. However, ribonuclease protection assay using cloned 5 UTR sequence revealed that the size of 5' UTR of erb B-2 mRNA which associate with transcription initiation site(s) in SNU-1 cells was longer than that in SNU-16. These results suggest that the increased erb B-2 protein synthesis rate possibly due to the redundant selection of transcription initiation might be a mechanism of erb B-2 overexpression in SNU-1 cells.
\end{abstract}

Keywords: c-erb B-2, oncogene, overexpression, stomach cancer

\section{Introduction}

Overexpression of erb B-2 protein is observed in 10$40 \%$ of the carcinoma of breast, ovary, lung and stomach cancer (Slamon et al., 1987; Weiner et al., 1990; Park et al., 1989). The mechanism of erb B-2 protein overexpression is known to be gene amplification and/or mRNA overexpression (Slamon et al., 1987; 1989). In addition to overexpression of erb B-2 at mRNA level, several reports suggested that post-transcriptional upregulation of c-erb B-2 might exist (Burhing et al., 1995; Oshima et al., 1995; Child et al., 1999). We earlier reported post-transcriptional overexpression of erb B-2 (Bae et al., 1993). In that study, erb B-2 protein level was upregulated without mRNA increase in SNU-1 and SNU-5 stomach cancer cells compared to SNU-16 or KATO III cells. Moreover, indifference in the overall protein synthesis rate between SNU-1 and SNU-16 cells suggest that participation of some post-transcriptional events in erb B-2 overexpression in these cell lines. Expression rate of erb B-2 protein in breast cancer was heterogeneous and does not correlated with erb B-2 mRNA expression rate.

Many oncogenes including c-erb B-2 are known to have 5' UTR with high GC content. 5' untranslated region of mRNA is known as one of the major cis-acting elements affecting protein translation rate. The structure of 5' UTR and existence of ATG codon in 5' UTR may affect ribosome scanning along mRNA (Hershey, 1991). So there is an open possibility that altered 5' UTR structure might affect expression level of such oncogenes.

In this study, we compared the synthesis and degradation rates of erb B-2 protein in SNU-1 and SNU-16 cells and found that erb B-2 protein overexpression in SNU-1 cells resulted from increased translation rate, not from altered protein degradation rate. There was no sequence difference in 5' UTR of c-erb B-2 mRNA between SNU-1 and SNU-16 cells. Instead, the length of 5' UTR in c-erb B-2 mRNA was varied between in SNU-1 and SNU-16 cells. These results suggested that selection of transcriptional initiation might be another mechanism of c-erb B-2 overexpression.

\section{Materials and Methods}

Cell culture, genomic DNA, total cellular RNA prepa- 


\section{ration}

All stomach cancer cells were cultivated in RPMI 1640 containing $10 \%$ FBS under $5 \% \mathrm{CO}_{2}$. Genomic DNA was extracted from cultured stomach cancer cells using proteinase $\mathrm{K}$ digestion followed by phenol extraction (Maniatis et al., 1989). Total cellular RNA was extracted using acid-guanidine-phenol method as described previously (Bae et al., 1996).

\section{Ribonuclease protection assay}

The genomic DNA fragment containing c-erb B-2 exon 1 (-1 to -323 region) was amplified using Taq DNA polymerase [Perkin-Elmer] using 7-deaza dGTP instead of dGTP for resolving possible stable secondary structure during PCR. For preparation of riboprobe, amplified cerb B-2 genomic DNA fragment was subcloned into pGEM3z and transcribed using T7 RNA polymerase in the presence of $\alpha\left[{ }^{32} \mathrm{P}\right]-\mathrm{CTP}$ in vitro. Total RNA $(25 \mu \mathrm{g})$ was hybridized at $50{ }^{\circ} \mathrm{C}$ for $16 \mathrm{~h}$ with ${ }^{32} \mathrm{P}$-labeled erb $\mathrm{B}$ 2 riboprobe and digested with RNase One [Promega] subsequently (Bae et al., 1996). The RNase One digestion products were separated through a $6 \%$ polyacrylamide gel containing $7 \mathrm{M}$ urea. The resulting gel was dried and exposed to Kodak X-Omat AR film at $-70^{\circ} \mathrm{C}$ for $18 \mathrm{~h}$.

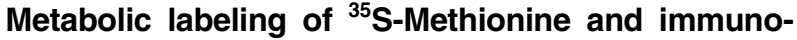 precipitation}

For short-term $(<4 \mathrm{~h})$ labeling, cells at mid-log phase were resuspened in Met-free RPMI [Gibco/BRL] containing $5 \%$ dialyzed FBS for 20 min and then incubated in the same medium containing $0.2 \mathrm{mCi}$ of ${ }^{35} \mathrm{~S}-\mathrm{Met}$ [Amersham]. For long-term labeling, Met-free RPMI containing 10\% normal RPMI 1640, 10\% FBS and 0.05 $\mathrm{mCi}$ of ${ }^{35} \mathrm{~S}-$ Met was used instead of Met-free RPMI containig $5 \%$ dialysed $\mathrm{FBS}$ and $0.2 \mathrm{mCi}$ of ${ }^{35} \mathrm{~S}$-Met. Cells labeled with ${ }^{35} \mathrm{~S}-M e t$ were resuspended in HNN buffer and lysed by passing through 23G needle. Each cell lysate was incubated for $4 \mathrm{~h}$ with anti-erb B-2 polysera at the dilution of $1: 80$ and then for $1 \mathrm{~h}$ with protein A-Sepharose [Amersham]. Antigen-antibody-protein A-sepharose complex was washed three times with HNN buffer and fractionated through $8 \%$ SDS-PAGE.

After polyacrylamide gel electrophoresis, Gels were fixed in $10 \%$ glacial acetic acid, $15 \%$ isopropyl alcohol and then soaked for 15-30 min in Amplify [Amersham], a fluorographic reagent. Gels were dried under vaccum and exposed to the preflashed films as described by Maniatis et al. (1989).

\section{Results}

\section{Half life of erb B-2 protein in SNU-1 cells}

Earlier report showed that expression level of erb B-2
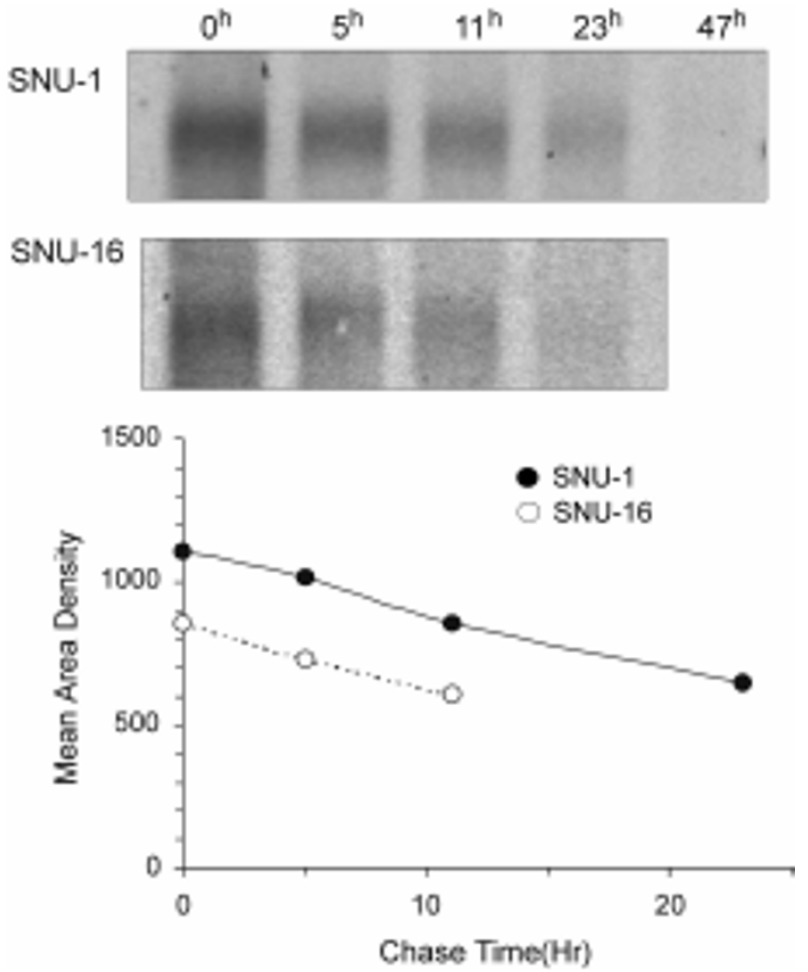

Figure 1. Degradation rate of erb B-2 gene product in SNU-1 cells and SNU-16 cells. SNU-1and SNU-16 cells at mid-log phase were pulse-labeled for $12 \mathrm{~h}$ and chased for $0,5,11,23,47 \mathrm{~h}$. Cell lysate at each time point was immunoprecipiated using anti-erb B-2 polysera and exposed to preflashed $\mathrm{X}$-Omat AR film for 6 days at $-70^{\circ} \mathrm{C}$ as described in methods. Erb B-2 protein bands were much weaker in SNU-16 cells due to lower level of erb B-2 protein expression. The densities of erb B-2 protein band were expressed as area densities and plotted to chase time.

protein in SNU-1 and SNU-5 cells 2 to 4 times higher than SNU-16 or KATO III cells without gene amplification or mRNA overexpression (Bae et al., 1993). Moreover, this overexpression of erb B-2 was gene-specific in that overall protein synthesis rate in SNU-1 or SNU-16 cells was similar. For exploring underlying mechanism, cellular metabolic state of erb B-2 protein were investigated by examining rate of degradation and synthesis in SNU-1 cells. The degradation rate of erb B-2 protein was measured by pusle-chase labeling followed by immunoprecipitation. The incorporation rate of ${ }^{35} \mathrm{~S}-$ methionine was similar in both cell lines. The specific activity (radioactivity/ $\mu \mathrm{g}$ protein) of the cell lysate at zero time was $3.9 \times 10^{4} \mathrm{cpm} / \mathrm{mg}$ in SNU-1 cells, $3.8 \times 10^{4}$ $\mathrm{cpm} / \mathrm{mg}$ in SNU-16 cells, respectively, which indicated that erb B-2 protein overexpression in SNU-1 cells was gene-specific.

As shown in Figure 1, the difference between the half life of erb B-2 protein in SNU-1 cells and that in SNU16 cells was not significantly different and not enough to explain the erb B-2 protein increase in SNU-1 cells although overall signal intensity was much less in SNU-16 cells due to the low level of erb B-2 protein expression, 
(a)

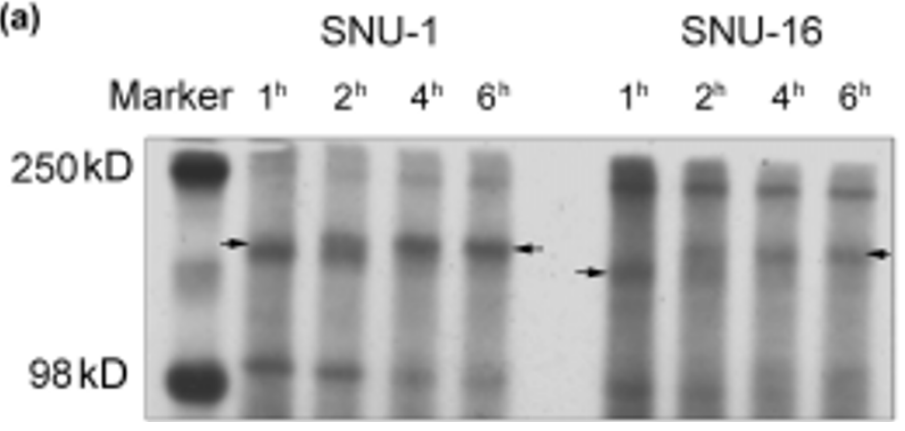

(b)

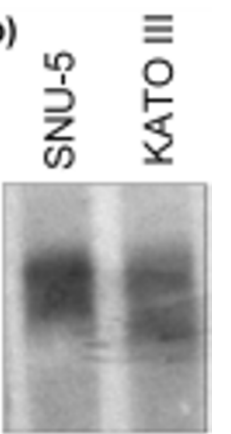

Figure 2. Synthesis of erb B-2 protein in SNU-1 and SNU-16 cells. (a) SNU-1 and SNU-16 cells were labeled for 1, 2, 4, and $6 \mathrm{~h}$ with $0.2 \mathrm{mCi} / \mathrm{ml}$ or $0.5 \mathrm{mCi} /$ $\mathrm{ml}$ of ${ }^{35} \mathrm{~S}$-Met, respectively. Cell lysate at each time point was immunoprecipitated using anti-erb B-2 polysera as described in methods. All immunoprecipitates were fractionated through the same gel and treated under same conditions for comparison between SNU-1 and SNU-16 cells. The fluorogram showed that main product of erb B-2 protein synthesized for the first $1 \mathrm{~h}$ period was immature form (170 kD, lower arrow). Fully processed erb B-2 protein (185 kD, upper arrow) was seen after $2 \mathrm{~h}$ time point. (b) SNU-5 and KATO III cells were labeled for a fixed time period, $2 \mathrm{~h}$ with ${ }^{35} \mathrm{~S}-\mathrm{Met}(0.2 \mathrm{mCi} / \mathrm{ml})$. All fluorograms were obtained after 2 days exposure to Kodak X-Omat AR film.

which left some difficulty in assessing the half life of erb B-2 protein in SNU-16 cells. This result suggested that erb B-2 protein half lives between these two cells was not significantly different and overexpression of erb B-2 protein in SNU-1 cells might be due to the other causes, i.e. protein synthesis rate.

\section{Synthesis rate of erb B-2 protein in SNU-1 cells}

Since there was very little difference in the rate of erb B2 protein degradation, the synthesis rate by measuring ${ }^{35} \mathrm{~S}$ incorporation rate into erb B-2 protein was carried out. A higher concentration of ${ }^{35} \mathrm{~S}-\mathrm{Met}(0.5 \mathrm{mCi} / \mathrm{ml})$ was used to facilitate the visualization of erb B-2 protein in SNU-16 cells than in SNU-1 cells $(0.2 \mathrm{mCi} / \mathrm{ml})$. As seen in Figure 2, erb B-2 protein labeled for $1 \mathrm{~h}$, representing newly synthesized protein, appeared to be slightly smaller molecule size than erb B-2 protein labeled for 2 or more $\mathrm{h}$. It is an interesting observation that a newly synthesized erb B-2 protein in both SNU-1 and SNU-16 cells appeared as a slightly smaller molecular size than matured protein where one can visualize the posttranslational processing (i.e. glycosylation) post one $\mathrm{h}$. Diffuse band pattern in erb B-2 protein labeled for $2 \mathrm{~h}$ also supported this idea. The amount of ${ }^{35} \mathrm{~S}$-labeled erb B-2 protein for $6 \mathrm{~h}$ was not increased compared to that for $4 \mathrm{~h}$ possibly by depletion of Met in the labeling medium. Since we used Met-free medium and dialyzed fetal bovine serum to maximize labeling efficiency, the concentration of Met in the labeling medium might not be sufficient for more than $2 \mathrm{~h}$.

The time course for post-translational modification was similar in both cells. However, when ${ }^{35} \mathrm{~S}$-labeled erb B2 protein for 1 or $2 \mathrm{~h}$ time period was compared, the amount of newly synthesized erb B-2 protein for the labeling period in SNU-1 cells was greater than that in SNU-16 cells even though higher amount of ${ }^{35} \mathrm{~S}$-Met was used for labeling SNU-16 cells (Figure 2a, see
Figure legend). Such results indicate that erb B-2 overexpression in SNU-1 cells was likely due to increased protein synthesis rate.

To confirm this hypothesis, the synthesis rate of erb B2 protein in SNU-5 cells, closely related to SNU-1 cells, was carried out under the same experimental conditions. Figure $2 \mathrm{~b}$ showed the amount of erb B-2 protein synthesized for $2 \mathrm{~h}$ in SNU- 5 cells was greater than that in KATO III cells, another stomach cancer cells showing simliar level of erb B-2 mRNA expression. Taken together, we concluded that erb B-2 overexpression in SNU- 1 and SNU- 5 cells resulted from increased rate of erb B-2 protein synthesis.

\section{Analysis of 5' untranslated region (UTR) of c-erb B-2 mRNA}

Since 5' UTR might affect the synthesis rate of protein and untranslated region of c-erb B-2 mRNA is known to contain high levels of GC, 5' UTR of c-erb B-2 mRNA in SNU-1 and SNU-16 cells were analyzed. We cloned and sequenced the genomic fragment $(-1$ to -323$)$ containing 5' UTR of c-erb B-2 mRNA using primers (upstream primer: GAGAAAGTGAAGCTGGGAGTTG, downstream primer: CCATGGTGCTCACTGCGGCT). We could amplify this region only when we used 7-deazadGTP, which resulted from extremely high GC content of this region. Five indifferent clones obtained from SNU-1 cells and 3 indifferent clones from SNU-16 (Figure 3) were sequenced. However, any mutations leading to either change in number of upstream ATG or significant change in GC content of 5' UTR were not detected. So we excluded the possible role of upstream ATG sequences in overexpression of erb B-2 protein in SNU-1 cells. We found 2 sequence differences (underlined sequence in Figure 3) compared to the published sequence (Gene bank accession number M16789). Since all the published erb B-2 promotor sequences are 


\section{GAG AAAGTGAAGC TGGGAgTtGC CACTCCCAGA -301 CTTGTTGgA TACAgTTGGA GGgGGCGAGC TGGGAgCGCA CTTGCTCCCA -251 cmorolpublished seguencel

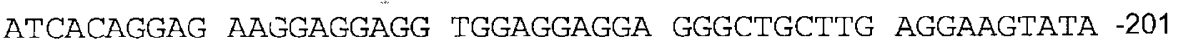 AGAATGAAGT TGTGAAGCTG AGA|TrTCCCCT CCATTGGGAC CGGAGAAACC -151 AGGGGAGCCC CCCGGGCAGC CGCGCGCCCC TTCCCACGGG GCCCTTTACT -101 GCGCCGCGCG CCCGGCCCCC ACCCCTCGCA GCACCCCCGG CCCCGCGCCC -51 TCCCAGCCGG GTCCAGCCGg AgCdATGGgG CCGGAGCCGC AGTGAGCACC -1 $\rightarrow$ Translation Initiation ATGGAGCTGG}

Figure 3. Sequence analysis of 5' UTR of c-erb B-2 mRNA in SNU-1 cells. The genomic DNA fragment containing 5' UTR was amplified using Taq DNA polymerase and sequenced (see text). Upstream ATG sequences were marked as clear box and the sequences different from previous publication were underlined. The sequence was the representative of 5 indifferent clones to rule out PCR error.

showing same sequences, these sequence differences might be related with usage of 7-deaza-dGTP in PCR. 7-deaza-dGTP can weaken hydrogen bond between $\mathrm{G}$ and $\mathrm{C}$ but might increase misincorporation rate in PCR.

The transcription initiation site was next examined by ribonuclease protection assay. As seen in Figure 4, three distinct fragments were protected from RNase digestion in all 3 stomach cancer cells (SNU-1, SNU-16, and KATO III), which suggest that there were three distinct transcription initiation sites. In both SNU-16 and

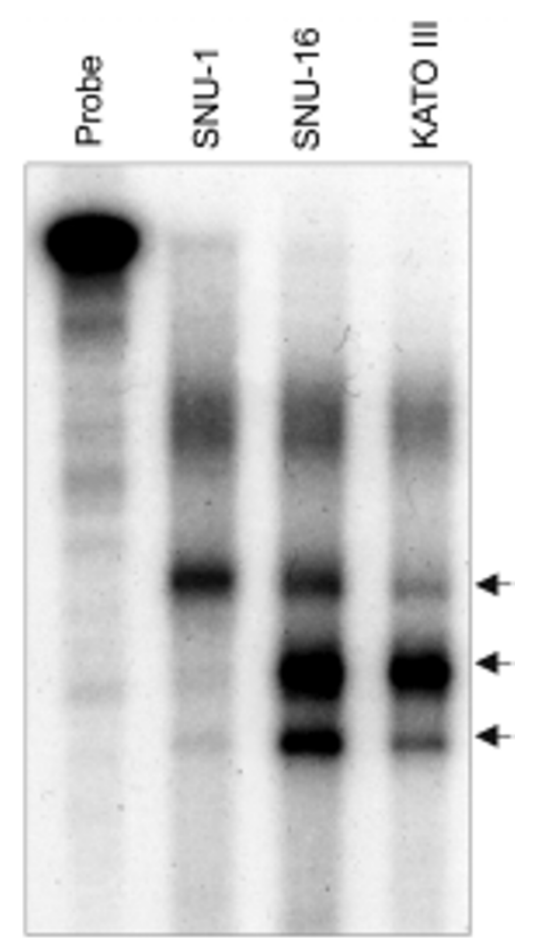

Figure 4. Anaysis of the length of 5' UTR of c-erb B-2 mRNA in SNU-1 and SNU-16 cells. The genomic DNA fragment was in vitro transcribed and subsequent RNA was used for ribonulcease protection assay. Autoradiogram was obtained using phosphoimage storage screen [Molecular Dynamics]. Three different fragments (arrows) were protected from RNase digestion.
KATO III cells, smaller two fragments were mainly protected while in SNU-1 cells, those two fragments were barely visible. Instead, a larger fragment was the mainly protected in SNU-1 cells.

These results suggest that the transcription initiation site of c-erb B-2 mRNA in SNU-1 cells was different from those in SNU-16 and KATO III cells and the upregulation mechanism of erb B-2 protein in SNU-1 cells might be due to longer 5' UTR. At the present, other genetic alterations such as translation initiation factors couldn't be excluded but redundant selection of transcription initiation sites might consider as a possible mechanism of erb B-2 overexpression in SNU-1 cells.

\section{Discussion}

c-erb B-2 oncogene is frequently overexpressed in primary human adenocarcinomas where overexpression of erb B-2 protein is related with poor prognosis. The molecular mechanism of erb B-2 overexpression in primary human cancer is known due to mRNA overexpression with/without gene amplification. However, the detailed mechanism of mRNA overexpression or gene amplification is still obscure. Moreover, in many cases, erb B-2 overexpression was detected by immunohistochemistry even though no c-erb B-2 mRNA overexpression was detected by northern blot anaylsis. In this study, we reported post-transcriptional overexpression mechanism of erb B-2 oncogene. As shown in Figure 2, synthesis rate of erb B-2 protein in SNU-1 cells was increased compared to SNU-16 cells while the half-life of erb B-2 protein was not significantly different (Figure $1)$. This enhanced protein synthesis rate was also observed in SNU-5 cells, showing erb B-2 protein overexpression without mRNA increase (Bae et al., 1993; 1996).

In an effort to find out underlying molecular mechanism of enhanced erb B-2 synthesis, we have targeted the initiation step as the rate controlling step in translation. 
The overall rate of protein elongation step in cells is reported to be near-maximum (5 amino acid/ribosome/ $\mathrm{sec}$ ) and has little effect on protein synthesis rate (Hershey, 1991). Among the factors affecting initiation step of protein synthesis, initiation factors and structure of mRNA are important (Hershey, 1991; Thach, 1992). Both the fact that erb B-2 mRNA has highly GC-rich 5' UTR (Figure 3) and the complexity of eukaryotic translation factors lead us to start our further study from exploring the possibility of genetic alterations in 5' UTR.

A possible presence of extra ATG upstream to translation initiation site which may make ribosome stall, or the altered secondary structure of 5' UTR which may block scanning ribosome along this region have also been considered (Child et al., 1999). We firstly, thought the latter possibility might be the case since the overall GC content of the genomic fragment (300 bp) including 5' UTR of c-erb B-2 mRNA was very high (67\%) which raised the possibilities of secondary structure. The fact that this region has 3 extra ATG sequences but 2 of those upstream ATG are located upstream to the known transcription initation site (Tal et al., 1987; Grooteclaes et al., 1994) suggests that ribosome stalling might not occur. In fact, we could exclude the role of upstream ATG since there was no mutation resulting in any change of upstream ATG in c-erb B-2 mRNA in SNU-1 cells. Moreover, we couldn't detect any sequence differences in the promoter region including exon1 between SNU-1 and SNU-16 cells. Instead, we found that 5' UTR length of c-erb B-2 mRNA in SNU-1 cells was longer than that in SNU-16 cells. This results might be confusing since longer 5' UTR with high GC content was likely to have more stable secondary structure. However, 5' UTR of cerb B-2 mRNA was relatively short, less than $200 \mathrm{bp}$, so shortening of 5' UTR didn't look like affecting the secondary structure greatly. Moreover, overall GC content was still high even though some shortening of 5' UTR occurred. Possible explanation of the effect of long 5' UTR was that ribosome entry to c-erb B-2 mRNA might be more facilitated. Other possible explanation would be the presence of other genetic alterations such as transacting initiation factors. At the present, we do not exclude the role of initiating factors, which is worth to be explored.

\section{Acknowledgement}

This research was supported partly by Hallym University Research Fund.

\section{References}

Bae, C.-D., Park, S.-E., Seong, Y.-S., Kimm, S.-W., Park, J.-
B. and Park, J.-G. (1993) The mechanism of c-erb B-2 gene product increase in stomach cancer cell lines. J. Kor. Med. Sci. 8: 153-159

Bae, C.-D., Lee, T.-H. and Park, J.-B. (1996) Quantitation of cerb B-2 mRNA by competitive reverse transcriptase-polymerase chain reaction. Mol. Cells 6: 101-107

Buhring, H. J., Sures, I., Jallal, B., Weiss, F. U., Busch, F. W., Ludwig, W. D., Handgretinger, R., Waller, H. D. and Ullrich, A. (1995) The receptor tyrosine kinase p185HER2 is expressed on a subset of B-lymphoid blasts from patients with acute lymphoblastic leukemia and chronic myelogenous leukemia. Blood 86: 1916-1923

Child, S. J., Miller, M. K. and Geballe, A. P. (1999) Translational control by an upstream open reading frame in the HER-2/neu transcript. J. Biol. Chem. 274: 24335-24341

Grooteclaes, M., Pasleau, F., Dijkmans, H., Berzi, P., Albert, A. and Winkler-Gol, R. (1994) The 6-kilobase c-erbB2 promoter contains positive and negative regulatory elements functional in human mammary cell lines. Cancer Res. 54: 4193-4199

Hershey, J. W. B. (1991) Translational control in mammalian cells. Ann. Rev. Biochem. 60: 717-755

Oshima, M., Weiss, L., Dougall, W. C., Greene, M. I. and Guroff, G. (1995) Down-regulation of c-neu receptors by nerve growth factor in PC12 cells. J. Neurochem. 65: 427-433

Park, J.-B., Rhim, J. S., Park, S.-C., Kimm, S.-W. and Kraus, M. H. (1989) Amplification, overexpression and rearrangement of the erb B-2 protooncogene in primary human stomach carcinomas. Cancer Res. 49: 6605-6609

Slamon, D. J., Clark, G. M., Wong, S. G., Levin, W. D., Ullrich, A. and McGuire, W. L. (1987) Human breast cancer: correlation of relapse and survival with amplification of the HER-2/neu oncogene. Science 235: 177-182

Slamon, D. J., Godophin, W., Jones, L. A., Wong, S. G., Keith, D. E., Levin, W. J., Stuart, S. G., Udove, J., Ullrich, A. and Press, M. F. (1989) Studies of the HER-2/neu proto-oncogene in human breast and ovarian cancer. Science 244: 707-712

Tal, M., King, C. R., Kraus, M. H., Ullrich, A., Schlessinger, J. and Givol, D. (1987) Human HER2 (neu) promoter: evidence for multiple mechanisms for transcriptional initiation. Mol. Cell. Biol. 7: 2597-2601

Thach, R. E. (1992) Cap recap: the involvement of elF-4F in regulating gene expression. Cell 68:177-80

Weiner, D. B., Nordberg, J., Robinson, R., Nowell, P. C., Gazdar, A., Greene, M. I., Williams, W. V., Cohen, J. A. and Kern, J. A. (1990) Expression of the neu gene-encoded protein $\left(\mathrm{p} 185^{\text {neu }}\right)$ in human non-small cell carcinomas of the lung. Cancer Res. 50: 421-425

Yamamoto, T., Ikawa, S., Akiyama, T., Semba, K., Nomura, N., miyajima, N., Saito, T. and Toyoshima, L. (1986) Similarity of protein encoded by the mammalian c-erb B-2 gene to epidermal growth factor receptor. Nature 319: 230-234 